

\title{
Impacts d'un retrait intense des rémanents sur la fertilité des sols forestiers et sur leur biodiversité
}

Emila Akroume

\section{To cite this version:}

Emila Akroume. Impacts d'un retrait intense des rémanents sur la fertilité des sols forestiers et sur leur biodiversité. Revue forestière française, 2014, Hors série 2014, pp.573-578. 10.4267/2042/56569 . hal-02636234

\section{HAL Id: hal-02636234 \\ https://hal.inrae.fr/hal-02636234}

Submitted on 27 May 2020

HAL is a multi-disciplinary open access archive for the deposit and dissemination of scientific research documents, whether they are published or not. The documents may come from teaching and research institutions in France or abroad, or from public or private research centers.
L'archive ouverte pluridisciplinaire HAL, est destinée au dépôt et à la diffusion de documents scientifiques de niveau recherche, publiés ou non, émanant des établissements d'enseignement et de recherche français ou étrangers, des laboratoires publics ou privés. 


\title{
IMPACTS D'UN RETRAIT INTENSE DES RÉMANENTS SUR LA FERTILITÉ DES SOLS FORESTIERS ET SUR LEUR BIODIVERSITÉ
}

\author{
Emila AKroume
}

\section{OBJECTIFS}

\section{Contexte général}

Dans le contexte énergétique actuel, les pouvoirs publics s'engagent à réduire la consommation des énergies fossiles et à développer le marché des énergies renouvelables afin de diminuer les émissions de $\mathrm{CO}_{2}$ et de faire face à la raréfaction des sources d'énergie fossile. Le Grenelle de l'environnement et la directive européenne 2009/28/CE ont fixé comme objectif d'atteindre $23 \%$ d'énergies renouvelables dans la consommation totale énergétique finale métropolitaine pour 2020 soit 20 Mtep d'énergies renouvelables en plus par rapport à 2006. La biomasse (issue de la forêt, de cultures énergétiques agricoles dédiées et de sous-produits d'agriculture) est la principale filière contributrice à la réalisation de cet objectif puisqu'elle devra constituer la moitié de ce dernier. Ceci devrait donc passer par une augmentation de la récolte de bois : une récolte supplémentaire de 10 millions de $\mathrm{m}^{3}$ était proposée par le Programme forestier national pour développer la filière bois énergie. Le Grenelle de l'environnement avait, quant à lui, préconisé une augmentation de 20 à $30 \%$ des prélèvements de bois entre 2012 et 2020 (Landmann et al., 2009). Cette mobilisation accrue de bois risque de conduire à une intensification des prélèvements et un changement des pratiques sylvicoles. Une des sources de biomasse forestière, encore peu en concurrence avec d'autres secteurs utilisateurs de bois, correspond à la part des menus bois et des rémanents d'exploitation. Ces derniers constituent une ressource potentielle non comptabilisée dans les volumes récoltés.

C'est dans ce contexte que se positionne ce sujet de thèse, portant sur l'impact du prélèvement des rémanents forestiers, sur la pérennité de la fertilité totale des sols et sur leur biodiversité.

\section{Objectifs et problématiques}

L'étude se développe sur deux volets parallèles : le premier volet porte sur les interactions entre les grands cycles biogéochimiques des sols forestiers (cycles du carbone et de l'azote) en se focalisant sur la litière et sur l'horizon organo-minéral. Le second volet du projet s'intéresse à l'impact de ces pratiques sylvicoles sur la faune et les communautés microbiennes du sol (bactériennes et fongiques), qui ont un rôle essentiel dans le fonctionnement des écosystèmes forestiers. On s'attardera en particulier sur les communautés fongiques saprotrophes responsables de la dégradation de la matière organique et sur les communautés de champignons mycorhiziens assurant les flux d'éléments minéraux vers l'arbre. 
Des études similaires sur les effets d'une intensification des récoltes forestières ont déjà été mises en place à l'étranger dans le cadre de réseaux de suivi des écosystèmes sur le long terme : le réseau CIFOR (Center for international forestry research) en forêt tropicale, le réseau nord-américain LTSP (long-term soil productivity) en zone boréale et subboréale, et un ensemble de sites en Fennoscandie en zone boréale.

Les branches et les feuilles constituent un stock important de minéralomasse, variable selon l'essence (Ranger et Bonneau, 1984). C'est pourquoi leur récolte conduit à une perte d'éléments susceptibles de retourner au sol et donc à une potentielle perte de fertilité des sols. Les réponses des écosystèmes sont différentes selon le contexte et les situations géographiques des études. Certains peuplements, surtout tropicaux, présentent à court terme des pertes brutales de biomasse microbienne et de fertilité chimique (Mendham et al., 2002). À l'inverse, les peuplements boréaux ou subboréaux semblent réagir beaucoup plus lentement ou plus faiblement. Ainsi, en Norvège et en Finlande, les peuplements ne montrent pas ou peu de réduction de croissance suite aux traitements "récolte d'arbres entiers", du moins avant 10 ans, ni même d'appauvrissement ou de modification des communautés de champignons saprophytes après 25 ans d'exploitation des rémanents (Allmér et al., 2009).

On note que les peuplements des zones tempérées sont moins bien représentés que les peuplements boréaux ou tropicaux dans les études d'impact. De plus, les aspects de fertilité chimique et de richesse biologique sont rarement étudiés conjointement. On peut supposer que les peuplements de plaine en zone tempérée se situeront dans une situation intermédiaire, avec des réponses échelonnées entre le court terme (perte d'éléments nutritifs, modification des assemblages microbiens) et le moyen ou long terme (impacts sur la croissance en volume, réactions aux aléas climatiques). Les réponses pourraient aussi dépendre fortement des sites, avec le risque d'observer des impacts sur la richesse chimique d'autant plus forts que le sol est initialement pauvre. Par ailleurs, l'exportation intense de biomasse (en particulier de litière) pourrait affecter de façon importante les communautés microbiennes, surtout les cortèges de champignons saprophytes et mycorhiziens inféodés à celle-ci. Cela pourrait se manifester par un déplacement vertical des communautés dans les 20 premiers centimètres du sol, une modification des assemblages (avec modification des abondances par taxon, remplacement de certains taxons par d'autres, et des fonctions qui leur sont allouées) ou par des pertes de richesse spécifique (disparition de taxons ou d'espèces et de la diversité fonctionnelle associée). Des perturbations de ces communautés microbiennes, et donc du fonctionnement biologique du sol, pourraient accentuer la perte de fertilité des sols les plus sensibles. À l'inverse, une éventuelle adaptation des assemblages microbiens pourrait permettre de maintenir l'activité biologique du sol, sur la base de complémentarités ou de redondances fonctionnelles.

On tentera d'apporter des réponses aux questions suivantes :

- Quels sont les effets d'un prélèvement accru de rémanents sur les flux biogéochimiques et sur la biodiversité sur sol?

- Quel est le niveau de résilience des écosystèmes forestiers face à l'intensification des prélèvements de biomasse?

- Quelles fonctions de l'écosystème peuvent être restaurées par des apports de cendres?

- Y a-t-il un seuil de non-retour à l'équilibre des flux ou des seuils de richesses microbiennes à partir desquels la restauration de l'équilibre n'est plus possible?

\section{ÉLABORATION DU RÉSEAU MOS : MATIÈRES ORGANIQUES DES SOLS}

Trois essences sont étudiées : le Hêtre (Fagus sylvatica L.), le Chêne (Quercus robur L. et Quercus petraea Liebl.) et le sapin de Douglas (Pseudotsuga menziensii Mirb.). L'idée est de travailler sur 
des peuplements représentatifs de la forêt française. Ces trois essences constituent une part importante de la production de bois d'œuvre et de construction en France. Le Hêtre et le Chêne seraient en effet les plus concernés par l'exploitation des rémanents à la suite des prélèvements de bois d'œuvre, tandis que le Douglas constitue une essence à fort potentiel dans un contexte de plantations à vocation de production de biomasse, en plus des usages actuels.

Un premier objectif est d'élaborer un réseau de 6 placettes expérimentales par essence, soit 18 sites au total, en forêt publique et privée. L'idée est de couvrir le gradient de fertilité et de conditions climatiques le plus large pour les stations de plaine. On souhaite se placer au maximum de l'accroissement courant annuel en volume. Cette première phase de développement du peuplement est intéressante pour les cycles biogéochimiques. En effet, elle correspond à la période de croissance optimale du peuplement, pour laquelle ses besoins en nutriments à prélever sont les plus importants. Quant aux micro-organismes (en particulier les champignons), les cortèges présentent une spécificité d'hôte importante au regard de l'essence dominante (Buée et al., 2011).

\section{Types de peuplements requis pour les expérimentations :}

- Peuplements de plaine purs, denses, avec peu de végétation au sol,

- Sur des stations homogènes et sans contrainte particulière (sont exclus les cas d'hydromorphie, de sols superficiels, de forte pente, etc.),

- Situés proche de l'accroissement courant annuel maximal : 20-30 ans pour le Douglas, et entre 30 et 60 ans pour les feuillus,

- Peuplements passés en éclaircie à l'hiver 2012-2013 ou 2013-2014.

Quatre traitements seront étudiés sur un site :

1. témoin (sylviculture habituelle),

2. retrait des rémanents seulement,

3. retrait des rémanents et retrait total de la litière,

4. compensation du retrait des rémanents par apport de cendres.

Nous avons prévu trois répétitions par modalité de traitement, soit au total 12 placettes de 16 ares, conduisant à des dispositifs expérimentaux de 2 hectares.

Une première phase de prospection a été menée entre mars et juillet 2013 sur les sites Chêne et Hêtre. Des échantillons de litière et des 20 premiers centimètres de sol (selon 3 profondeurs : $0-5 \mathrm{~cm}$, $5-10 \mathrm{~cm}$ et $10-20 \mathrm{~cm}$ ) ont été prélevés systématiquement sur les 2 hectares de chaque site, selon un maillage de $20 \mathrm{~m}$ par $20 \mathrm{~m}$. Ces échantillons ont été tamisés à $5 \mathrm{~mm}$, séchés, et broyés pour leur conditionnement et les différentes analyses. Ils ont été d'abord traités par scanner NIRS/MIRS(1) afin de déterminer les hétérogénéités inter- et intra-sites et de dégager des groupes de similarité entre les différents blocs. La spectrométrie infrarouge repose sur le principe de réflexion d'un rayon infrarouge sur la surface de l'échantillon de sol. Le signal est renvoyé sous forme d'un spectre unique, propre à chaque échantillon selon ses propriétés physiques et chimiques. Les résultats des analyses NIRS/MIRS font l'objet d'un traitement statistique par des méthodes de régressions multiples (ACP) pour chaque horizon de sol étudié. La comparaison des spectres de réponse pour chacun des échantillons prélevés donne alors accès rapidement et de façon complète aux éventuels gradients ou hétérogénéités spatiales existantes sur un site. Les coordonnées des ACP sont cartographiées pour établir des cartes de variation des propriétés du sol pour chaque site (figure 1, p. 576). La disposition des traitements sur ces différents blocs est alors tirée aléatoirement, de façon à tenir compte et s'affranchir de la variabilité du sol et du peuplement. Les données de cette phase préliminaire permettront de déduire des corrélations entre signaux infrarouges et caractéristiques des sols. Des analyses chimiques seront effectuées par la suite.

(1) Near infra-red spectrometry/mid infra-red spectrometry. 
FIGURE 1

\section{EXEMPLE DE CARTE DE VARIABILITÉ DU SOL OBTENUE À PARTIR DES DONNÉES NIRS \\ SUR LE SITE DE DARNEY (VOSGES)}

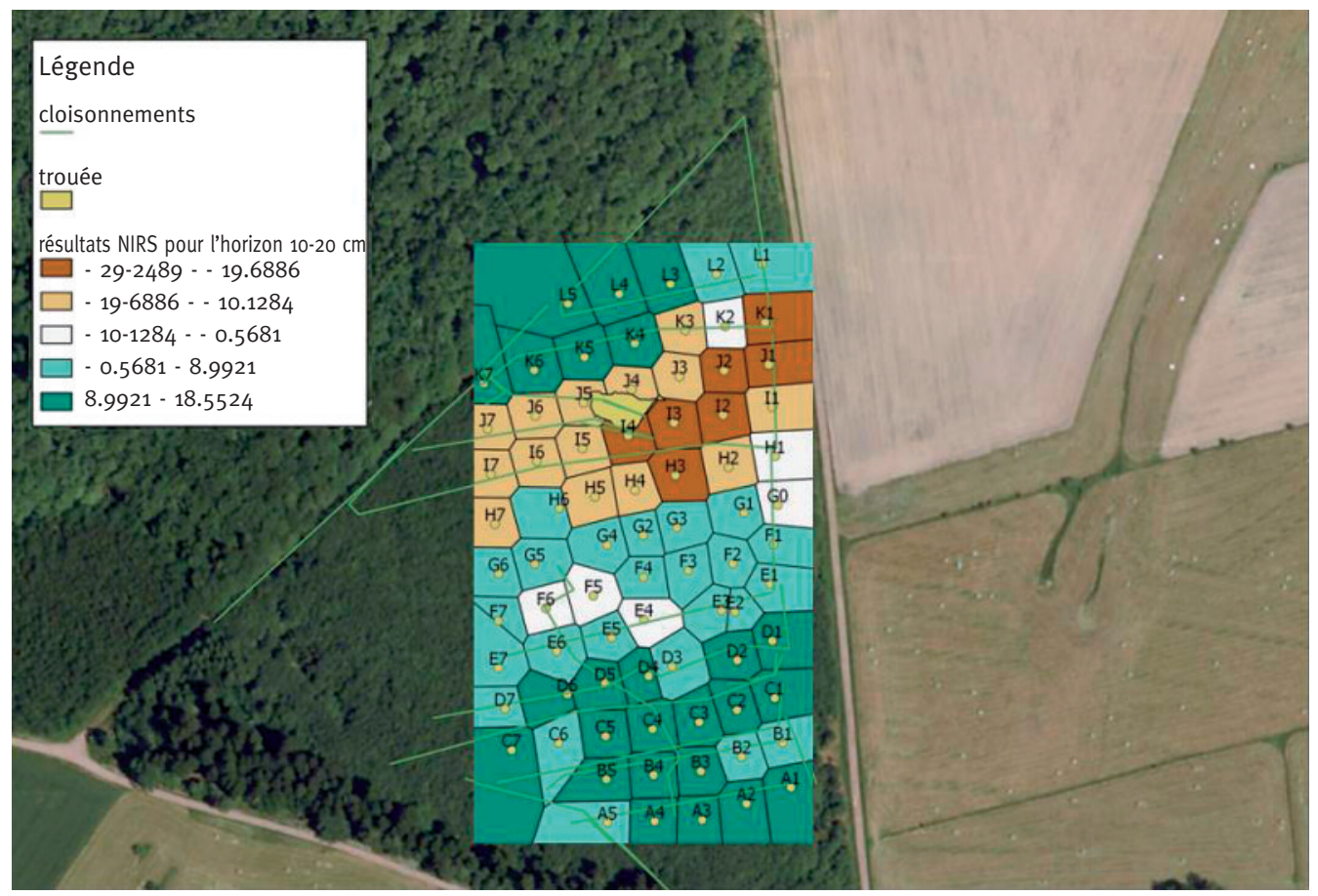

Des analyses de biologie moléculaire sont menées sur ces échantillons pour quantifier et identifier la diversité fongique et bactérienne des différents sites avant la mise en place des traitements. La problématique biodiversité sera abordée selon plusieurs aspects complémentaires mettant en application les approches de métagénomique par séquençage haut débit des marqueurs taxonomiques bactériens (région 16S) et fongiques (région ITS). La diversité sera estimée à partir de l'étude de la structure des communautés fongiques et bactériennes. Par ailleurs, les proportions eucaryotes/ procaryotes pourront être envisagées par PCR quantitative sur les régions $16 \mathrm{~S}$ et 18S, donnant un aperçu de la richesse et de l'abondance relative en taxons des différents sites étudiés, associé à un aspect patrimonial. Le volet fonctionnement des écosystèmes sera abordé à travers des mesures d'activité enzymatique dans les solutions de sol en se focalisant sur le métabolisme des éléments principaux : C, N, P, S. On testera principalement les activités de dégradation de la cellulose, de la lignine, des activités protéolytiques et de la mobilisation du phosphore, majoritairement portées par les espèces fongiques.

Le réseau MOS a pour vocation l'étude des effets sur le long terme (minimum 20 ans) de manipulations de la matière organique. En effet, la complexité des écosystèmes forestiers nécessite un suivi sur plusieurs échelles de temps. Certaines réponses sont souvent observables sur le court terme comme des modifications de minéralisation de l'azote, remarquables deux ans après perturbation en forêt tropicale, ou au cours des cinq premières années après traitement dans les cas de la minéralisation du carbone et d'azote et des stocks d'éléments minéraux, en forêt boréale et tempérée (Smolander et al., 2010). Les communautés microbiennes peuvent réagir assez rapidement aux perturbations telles qu'une éclaircie. Sur des sols sensibles tropicaux, une exportation intense des 
rémanents a provoqué une diminution de la biomasse microbienne un an après la manipulation (Mendham et al., 2002).

En revanche, les questions de résilience de l'écosystème requièrent des études sur le plus long terme. En forêt tempérée ou boréale (réseau LTSP Amérique du Nord), il a été montré que les exportations de menus bois impliquaient des modifications au sein des communautés microbiennes: 10 à 15 ans après perturbation, les communautés demeurent encore fortement affectées (Hartmann et al, 2012), ainsi que la disponibilité en éléments nutritifs (Sayers, 2006). Il semble donc nécessaire de suivre ces écosystèmes sur une période au-delà de 20 ans quant à l'éventuelle résilience des sites perturbés. Enfin, une des missions du réseau MOS sera d'identifier des impacts potentiels des manipulations de la matière organique sur les aspects croissance/production des peuplements forestiers. Certains peuplements à croissance rapide ont présenté des baisses de production en moins de 7 années (Laclau et al., 2010). Mais en zone boréale ou tempérée, sur des essences à croissance plus lente, menées avec des rotations plus longues, la plupart des peuplements qui sont impactés (récolte des rémanents ou raclage de la litière) ne réagissent qu'à partir de 15-20 ans après intervention (Sayers, 2006).

\section{APPORTS TECHNIQUES ET SYLVICOLES}

Le réseau MOS est mis en place et géré par l'unité BEF du Centre INRA de Nancy-Lorraine, en collaboration avec le département Recherche \& Développement de l'ONF.

Dans un premier temps, les résultats obtenus pourront compléter les connaissances sur l'impact du prélèvement des rémanents, notamment issues des travaux de l'ADEME (Cacot et al., 2006 ; Achat et al., 2013). Ils apporteraient une continuité et un approfondissement sur les questions de biogéochimie et de fertilisations compensatoires des sols forestiers tout en intégrant la dimension biodiversité fongique et bactérienne. Le réseau MOS est complémentaire du réseau RENECOFOR. Il s'agit ici d'effectuer des manipulations anticipant des évolutions possibles de la sylviculture française afin d'identifier des éventuels impacts, de les quantifier et de proposer des pistes de gestion forestière. Enfin, le réseau MOS constitue un support conséquent pour le suivi sur le long terme des questions de fertilité et de productivité des écosystèmes forestiers tempérés associées aux mesures de diversité microbienne, permettant ainsi de compléter les données apportées par les réseaux existant en forêt tropicale et boréale.

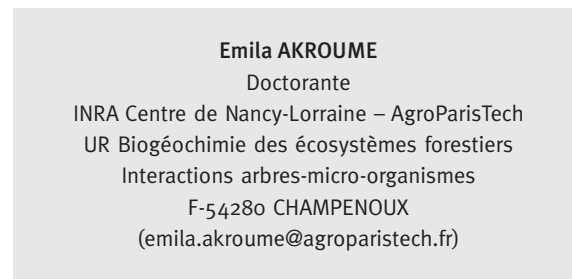

\section{Encadrants :}

Marc BUÉE - UMR Université de Lorraine-INRA Interactions arbres micro-organismes, Centre INRA de NancyLorraine - F-54280 CHAMPENOUX (marc.buee@nancy.inra.fr) 


\section{BIBLIOGRAPHIE}

ACHAT (D.), AUGUSTO (L.), DELEUZE (C.), LANDMANN (G.), POUSSE (N.), RANGER (J.). - Analyse bibliographique des impacts du prélèvement des rémanents forestiers sur la fertilité des sols et la croissance des peuplements. Rapport RESOBIO «Gestion des rémanents forestiers : préservation des sols et de la biodiversité ». GIP-ECOFOR - ADEME, 2013. - 122 p.

ALLMÉR (J.), STENLID (J.), DAHLBERG (A.). - Logging-residue extraction does not reduce the diversity of litterlayer saprotrophic fungi in three Swedish coniferous stands after 25 years. - Canadian Journal of Forest Research, 39, 2009, pp. 1737-1748.

BUÉE (M.), MAURICE (J.-P.), ZELLER (B.), ANDRIANARISOA (S.), RANGER (J.), COURTECUISSE (R.), MARÇAIS (B.), LE TACON (F.). - Influence of tree species on richness and diversity of epigeous fungal communities in a French temperate forest stand. - Fungal Ecology, 4, 2011, pp. 22-31.

CACOT (E.), NEISNER (N.), CHARNET (F.), LEON (P.), NICOLLEAU (C.), RANGER (J.). - La Récolte raisonnée des rémanents en forêt. Guide pratique. - Angers : ADEME Éditions, 2006. - 36 p.

HARTMANN (M.), HOWES (C.G.), VANINSBERGHE (D.), YU (H.), BACHAR (D.), CHRISTEN (R.), NILSSON (R.H.), HALLAM (S.J.), MOHN (W.W.). - Significant and persistent impact of timber harvesting on soil microbial communities in Northern coniferous forests. - The ISME Journal, 6, 2012, pp. 2199-2218.

LACLAU (J-P.), LEVILLAIN (J.), DELEPORTE (P.), NZILA (J.D.), BOUILLET (J.-P.), SAINT-ANDRÉ (L.), VERSINI (A.), MARESCHAL (L.), NOUVELLON (Y.), THONGO M'BOU (A.), RANGER (J.). - Organic residue mass at planting is an excellent predictor of tree growth in Eucalyptus plantations established on a sandy tropical soil. - Forest Ecology and Management, 260, 2010, pp. 2148-2159.

LANDMANN (G.), GOSSELIN (F.), BONHÊME (I.) (coord.). - Bio 2. Biomasse et diversité forestière. Augmentation de l'utilisation de la biomasse forestière : implications pour la biodiversité et les ressources naturelles. Paris : MEEDDM ; ECOFOR, 2009. - $211 \mathrm{p}$.

MENDHAM (D.), SANKARAN (K.), O'CONNELL (A.), GROVE (T.). - Eucalyptus globulus harvest residue management effects on soil carbon and microbial biomass at 1 and 5 years after plantation establishment. - Soil Biology and Biochemistry, 34, 2002, pp. 1903-1912.

RANGER (J.), BONNEAU (M.). - Effets prévisibles de l'intensification de la production et des récoltes sur la fertilité des sols de forêt. Le cycle biologique en forêt. - Revue forestière française, vol. XXXVI, n² 2, 1984, pp. 93-112.

SAYERS (E.J.). - Using experimental manipulation to assess the roles of leaf litter in the functioning of forest ecosystems. - Biological Reviews, $\mathrm{n}^{\circ}$ 81, 2006, pp. 1-31.

SMOLANDER (A.), KITUNEN (V.), TAMMINEN (P.), KUKKOLA (M.). - Removal of logging residue in Norway spruce thinning stands: Long-term changes in organic layer properties. - Soil Biology and Biochemistry, vol. 42, $n^{\circ} 8,2010$, pp. $1222-1228$.

\section{ImPACTS D'UN RETRAIT INTENSE DES RÉmANENTS SUR LA FERTILITE DES SOLS FORESTIERS ET SUR LEUR BIODIUERSITE [Résumé]}

Cette thèse porte sur l'impact du prélèvement des rémanents sur la fertilité et la biodiversité des sols forestiers, dans un contexte énergétique caractérisé par une volonté conjointe de réduire la consommation des énergies fossiles et de développer le marché des énergies renouvelables. Cette étude vise à aborder deux aspects complémentaires: les interactions entre les cycles biogéochimiques du carbone et de l'azote dans les écosystèmes forestiers, et l'impact des pratiques sylvicoles sur les communautés bactériennes et fongiques du sol. Elle a pour support un réseau de 18 placettes de suivi sur le long terme de l'effet de la manipulation des rémanents pour trois essences (Chêne, Hêtre et Douglas), le réseau matières organiques des sols (MOS). L'essentiel de cette thèse consiste en l'élaboration et la caractérisation initiale du réseau MOS.

\section{IMPACT OF INTENSIUE RESIDUE REMOUAL ON THE FERTILITY OF FOREST SOILS AND ON THEIR BIODIUERSITY [Abstract]}

This PhD deals with the impact of logging residues removal on forest soil fertility and biodiversity. It takes place into an energy environment characterised by a policy of reducing fossil energy consumption and developing renewable energies market, particularly the fuel wood sector. This study focuses on two aspects: the interactions between carbon and nitrogen cycling in forest soils and the impacts of these types of forest management on soil microbial and fungal communities. It is conducted on an 18-site long-term monitoring network called Matières Organiques des Sols (MOS). Three tree species are studied: oak, beech and Douglas fir. The PhD mostly consists in devising and establishing the baseline characteristics of the MOS network. 